\title{
Numerical modeling of the low cycle fatigue: effect of manufacturing imperfections caused by machining process
}

\author{
Ikram Abarkan $^{1 *}$, Abdellatif Khamlichi ${ }^{2}$, and Rabee Shamass ${ }^{3}$ \\ ${ }^{1}$ Department of Physics, Faculty of Sciences, Abdelmalek Essaadi University, 93002 Tetouan, Morocco \\ ${ }^{2}$ Department of Industrial and Civil Sciences and Technologies, National School of Applied Sciences, \\ Abdelmalek Essaadi University, 93030 Tetouan, Morocco \\ ${ }^{3}$ Division of Civil and Building Services Engineering, School of the Built Environment and \\ Architecture, London South Bank University, SE1 0AA London, United Kingdom
}

\begin{abstract}
The majority of mechanical components in nuclear power plants must be designed to withstand extreme cyclic loading conditions. In fact, when these components are subjected to low cycle fatigue, machining imperfections are considered one of the most significant factors limiting their service life. In the present work, using finite element analysis, a methodology has been suggested to predict the fatigue life of cylindrical parts made of $316 \mathrm{SS}$, at ambient temperature, under nominal strain amplitude ranging from \pm 0.5 to $\pm 1.2 \%$ with various surface roughness conditions. Two different multiaxial strain-life criteria have been considered to estimate the fatigue life, namely Brown-Miller and maximum shear strain. The comparison between the predicted and the experimental fatigue lifetimes has revealed that the adopted multiaxial strain life criteria can successfully estimate the fatigue life of $316 \mathrm{SS}$ grade under uniaxial loading conditions. Furthermore, it has been found that the fatigue life decreases as the surface roughness average value increases, which indicates that surface regularities have a significant impact on low cycle fatigue life. Therefore, the proposed methodology is found to be capable of assessing the impact of surface roughness on the fatigue life of this specific steel in the low cycle fatigue regime.
\end{abstract}

\section{Introduction}

With the increasing global demand for energy generation, mechanical components, in the nuclear power plant industries, are expected to operate at extreme temperature and pressure loading patterns. Therefore, Low Cycle Fatigue (LCF) is one of the major problems that cause the failure of these components. In fact, fatigue cracks are produced due to cumulative fatigue damage that occurs gradually even if the applied stress is much less than the elastic limit of the material [1]. Usually, a high number of cycles are needed to produce fatigue failure under low amplitudes of cyclic forces. Nevertheless, a fatigue failure may occur with a lower number of cycles when a material undergoes higher amplitudes of applied stresses.

* Corresponding author: aberkan.ikraam@,gmail.com 
Over the past several years, there has been a great concern among researchers to find reliable tools that can accurately predict the fatigue life of mechanical parts, even more, to increase their durability. The obsession with fatigue topics dates back to the $1800 \mathrm{~s}$, when Wöhler [2] introduced the commonly known S-N curve, which relates the stress amplitude to the number of cycles to failure, in the high cycle fatigue regime. Throughout the years, many empirical methods have been developed to estimate the fatigue life, for various type of materials under different cyclic loading conditions. In particular, Basquin [3] proposed a linear equation, in the log-log scale, that links the stress amplitude with the number of reversals to failure in the high cycle fatigue regime. Similarly, Coffin and Manson [4-5] suggested a linear equation, in the logarithmic scale, for low cycle fatigue regime that correlates the plastic strain amplitude to the number of reversals to failure. Besides, many international standards have been published to give a thorough fatigue design guidance. The Eurocode 3 [6] is one good example to mention. Most recently, numerical methods have become widely used to predict the low cycle fatigue in the engineering field because, in addition to their accuracy, they are more affordable and less laborious than the experimental assessment.

During service, there are too many factors that affect the fatigue life of a mechanical components. Among them are the material properties, the presence of notch in the geometry, the surface quality, temperature, and environment effect. The surface irregularities is the only parameter that can be controlled during the manufacturing process, while the environmental effect and temperature are unpredictable, and notch geometry such as holes are unavoidable in most of the machine components. Therefore, to enhance the fatigue life, it is important to understand the behavior of the material when it is subjected to cyclic loading under various surface roughness [7].

The 316 Austenitic Stainless Steel (SS) is the most widely used material for engineering components in the nuclear power plant industries [8], such as in the nuclear reactors, owing to outstanding mechanical and low cycle fatigue properties, as well as good anti-corrosion resistance, at low and high temperatures [8-9].

Since the Coffin-Manson [4-5] equation does not account for the influence of surface irregularities on fatigue life, and the localized multiaxial states of stress surrounding the defects. A different methodology is used in the present paper; the cyclic stress-strain behavior has been initially evaluated, using finite element analysis (FEA) in the ABAQUS-Standard software [10], for dog-bone shaped specimens made of 316 SS and subjected to uniaxial low cycle fatigue loading, at room temperature. Two different fatigue life methods have been considered to estimate the fatigue life of this steel, namely Brown-Miller and maximum shear strain multiaxial strain-life criteria [11-12], and Morrow mean stress correction [13] has been used to account for the influence of mean stress on fatigue life. The numerically obtained fatigue lives have been compared against the experimental data provided in [14]. Finally, by using Fe-Safe software [15], the effect of the surface imperfection on the cyclic life has been studied for various intervals of surface roughness average and applied strain amplitude levels.

\section{Numerical Modeling}

In order to obtain the cyclic stress-strain values for each applied strain amplitude level, the finite element analysis has been conducted on the ABAQUS software [10], and the output data have been imported into the Fe-Safe software [15] to estimate the fatigue life of the specimens under study. Figure 1 illustrates the followed instructions for the numerical simulation. 


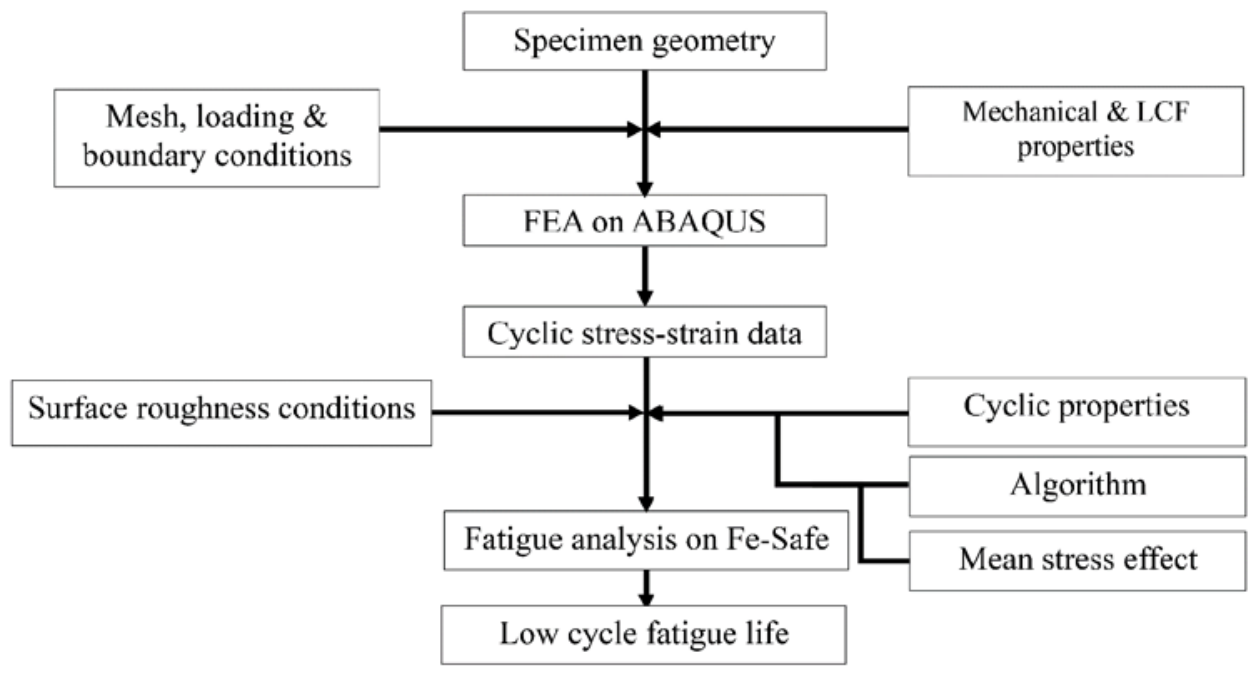

Fig. 1. Schematic representation of the followed instructions for the numerical modelling.

\subsection{Specimen geometry}

The low cycle fatigue simulation has been carried out on smooth dog-bone shaped specimens, which were employed in the LCF test carried out by Joadder et al. [14], with gauge length and gauge diameter of 15 and $7.5 \mathrm{~mm}$, respectively. Figure 2 depicts the shape and dimensions, in millimeters, of the specimens under investigation.

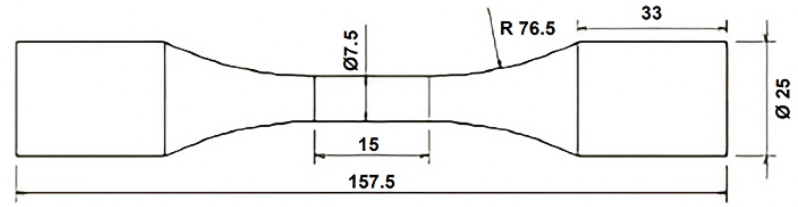

Fig. 2. Low cycle fatigue specimen's geometry, all dimensions are in mm.

\subsection{Material properties}

The material selected for this study is the low carbon grade of 316 austenitic stainless steel, a typically used for reactors in the nuclear power plants. The elastic as well as the plastic properties i.e. the non-linear isotropic and kinematic cyclic hardening parameters [16] have been incorporated into ABAQUS Software [10] to determine the hysteresis loops for each nominal strain amplitude. The material properties of $316 \mathrm{SS}$ are listed in Table 1.

Table 1. Mechanical and hardening properties of 316 SS at ambient temperature [14].

\begin{tabular}{|c|c|c|c|c|c|c|}
\hline $\begin{array}{c}\text { Elastic } \\
\text { modulus }\end{array}$ & $\begin{array}{c}\text { Poisson } \\
\text { ratio }\end{array}$ & $\begin{array}{c}\text { Yield } \\
\text { strength }\end{array}$ & \multicolumn{2}{|c|}{$\begin{array}{c}\text { Kinematic cyclic } \\
\text { hardening parameters }\end{array}$} & \multicolumn{2}{|c|}{$\begin{array}{c}\text { Isotropic cyclic hardening } \\
\text { parameters }\end{array}$} \\
\hline $\boldsymbol{E}(\boldsymbol{G P a})$ & $\boldsymbol{\vartheta}_{\boldsymbol{e}}$ & $\boldsymbol{\sigma}_{\boldsymbol{y}}(\boldsymbol{M P a})$ & $\boldsymbol{C}(\boldsymbol{M P a})$ & $\boldsymbol{\gamma}$ & $\boldsymbol{Q}_{\infty}(\boldsymbol{M P a})$ & $\boldsymbol{b}$ \\
\hline 200 & 0.3 & 220 & 35000 & 430 & 142 & 11.7 \\
\hline
\end{tabular}




\subsection{Mesh, Loading \& Boundary Conditions}

In the ABAQUS Software [10], the specimens have been subjected to five different nominal strain amplitudes, namely $\pm 0.5, \pm 0.6, \pm 0.75, \pm 1.0$, and $\pm 1.2 \%$. The C3D8R element type has been selected in this analysis. The numerical model has been fixed on one end while prescribed displacement, in the form of fully reversed triangular tension-compression load (i.e. $\mathrm{R}_{\varepsilon}=-1$ ), has been applied on the other end along the longitudinal direction. Figure 3 represents the applied loading and the boundary conditions of the FE model. Besides, a mesh convergence study has been performed on ABAQUS software [10] and a fine mesh of 1.5 $\mathrm{mm}$ has been applied to the 3D-part, as shown in Figure 4.

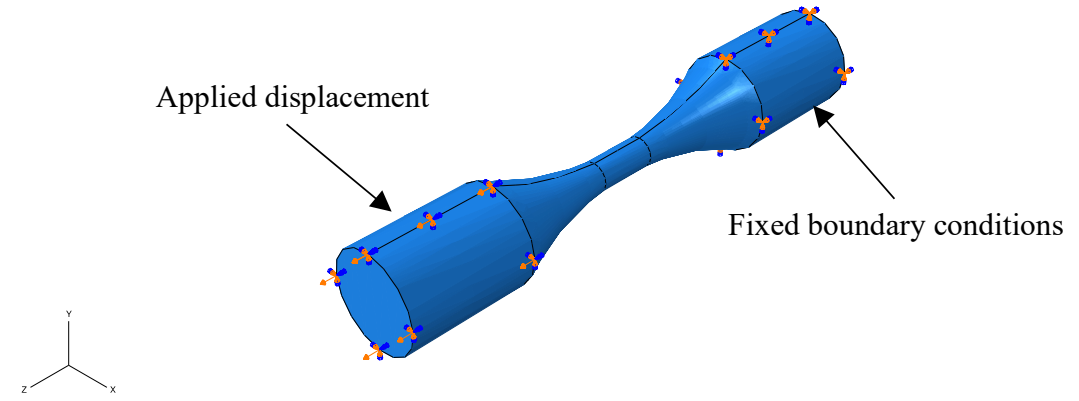

Fig. 3. The applied load and boundary conditions of the LCF simulation.

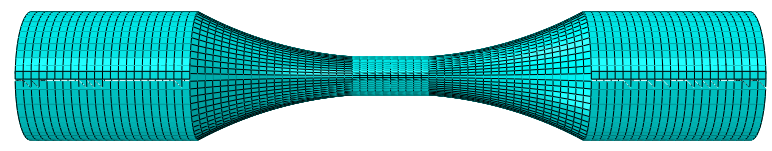

Fig. 4. The tridimentional meshed part.

\subsection{Fatigue Analysis on Fe-Safe}

The cyclic stress-strain data for different strain amplitudes have been imported from ABAQUS [10] into Fe-safe [15] fatigue dedicated software. The nodal elements of the 3D numerical model have been chosen as they are on the surface and the elastic-plastic block has been created to account for the material's elastic-plastic condition. The cyclic properties of 316 SS given in the Table 2 have been adopted. Since the presence of surface imperfections are commonly described as small notches on the surface [9], therefore even if the applied loading is uniaxial, localized multiaxial states of stress is expected around these defects. As a result, the multiaxial Brown-Miller and maximum shear strain criteria with Morrow mean stress correction [11-13] presented in (1) and (2) respectively have been selected as algorithms in FE-Safe [15], to predict the low cycle fatigue life. Additionally, the average of surface roughness has been introduced in the software to consider the effect of imperfections due to machining Process on the component's durability.

$$
\begin{gathered}
\frac{\Delta \gamma_{\max }}{2}+\frac{\Delta \varepsilon_{n}}{2}=1.65 \frac{\sigma_{f}^{\prime}-\sigma_{m}}{E}\left(2 N_{f}\right)^{b}+1.75 \varepsilon_{f}^{\prime}\left(2 N_{f}\right)^{c} \\
\frac{\Delta \gamma_{\max }}{2}=1.3 \frac{\sigma_{f}^{\prime}-\sigma_{m}}{E}\left(2 N_{f}\right)^{b}+1.5 \varepsilon_{f}^{\prime}\left(2 N_{f}\right)^{c}
\end{gathered}
$$


Where $\Delta \gamma_{\max }$ is the maximum shear strain range, $\Delta \varepsilon_{n}$ is the normal strain range, $2 N_{f}$ is the number of reversals to failure, $b$ is the fatigue strength exponent, $\sigma_{f}^{\prime}$ is the fatigue strength coefficient, $c$ is the fatigue ductility exponent, $\varepsilon_{f}^{\prime}$ is the fatigue ductility coefficient, $E$ is the Young's modulus, and $\sigma_{m}$ is the mean stress.

Table 2. Cyclic properties of 316 SS [14].

\begin{tabular}{|c|c|c|c|c|c|}
\hline \multicolumn{2}{|c|}{$\begin{array}{c}\text { Cyclic stress-strain curve } \\
\text { coefficients }\end{array}$} & \multicolumn{2}{c|}{ Basquin coefficients } & \multicolumn{2}{c|}{$\begin{array}{c}\text { Coffin-Manson } \\
\text { coefficients }\end{array}$} \\
\hline $\boldsymbol{K}^{\prime}(\boldsymbol{M P a})$ & $\boldsymbol{n}$ & $\boldsymbol{\sigma}_{\boldsymbol{f}}^{\prime}(\boldsymbol{M P a})$ & $\boldsymbol{b}$ & $\boldsymbol{\varepsilon}_{\boldsymbol{f}}^{\prime}$ & $\boldsymbol{c}$ \\
\hline 2854 & 0.378 & 1712 & -0.2092 & 0.1357 & -0.4503 \\
\hline
\end{tabular}

\section{Results and discussion}

The cyclic stress-strain results obtained from ABAQUS have been compared with the experimental ones found by Joadder et al. [14]. Moreover, the fatigue lives obtained numerically from Fe-Safe software [15], using the aforementioned multiaxial strain-life equations [11-13], have been compared with those obtained experimentally in [14]. Finally, the effect of surface imperfections due to the machining process has been analyzed, for various surface roughness average values and strain amplitude levels.

\subsection{Cyclic stress-strain behavior}

The cyclic stress-strain results have been obtained for different applied strain amplitude levels. A comparison between the experimental and numerical hysteresis loops is made. For brevity, only the cyclic stress-strains results under a nominal strain amplitude of \pm 0.5 are presented in Figure 5. One can observe, from this figure, that the numerically obtained first cycle hysteresis loop is in good agreement with the experimental one. Hence, the suggested numerical model is reliable, and can successfully predict the cyclic behavior of $316 \mathrm{SS}$, at room temperature.

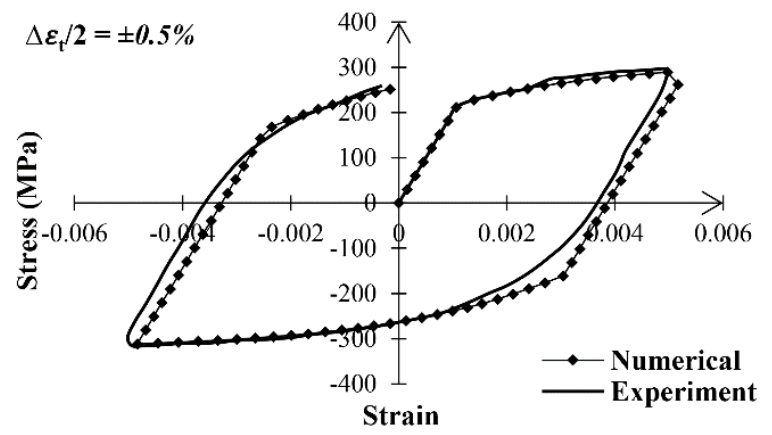

Fig. 5. A comparison between the experimentally and the numerically obtained first cycle hysteresis loops for a nominal strain amplitude of $\pm 0.5 \%$.

\subsection{Low cycle fatigue life assessment}

To examine the capability of multiaxial low cycle fatigue strain-life equations in predicting the fatigue life of smooth specimens subjected to fully reversed uniaxial cyclic tensioncompression loadings, Brown-Miller and maximum shear strain criteria both with Morrow 
mean stress correction [11-13] have been adopted in the Fe-Safe [15]. Since the surface texture is ground for the specimens used in the experiment [14], the average surface roughness value has been considered to be less than $0.25 \mu \mathrm{m}$, i.e. $\mathrm{R}_{\mathrm{a}} \leq 0.25 \mu \mathrm{m}$ [17].

The numerically obtained low cycle fatigue life for each nominal strain amplitude level, using the previously mentioned fatigue life methods [11-13], are plotted in the logarithmic scale against the experimental data, as illustrated in Figure 6. It can be observed that the estimated low cycle fatigue lives are generally conservative, which is evident that BrownMiller and maximum shear strain multiaxial criteria can safely predict the low cycle fatigue life of cylindrical specimens made of $316 \mathrm{SS}$, at room temperature, and subjected to fully reversed uniaxial tension-compression cyclic loadings. Moreover, the maximum shear strain equation with Morrow mean stress [12-13] is found to provide LCF life estimation more inline with the test results than those predicted by Brown and Miller equation with Morrow mean stress effect $[11,13]$. Table 3 gives the percentage relative error between the calculated and experimental low cycle fatigue life for both methods. The relative error from the maximum shear strain criterion ranges from -24.84 to $1.96 \%$ while the error from BrownMiller criterion ranges from -41.50 to $-21.17 \%$. For instance, when the nominal strain amplitude is $\pm 0.6 \%$, the resulted relative errors from Brown-Miller, and maximum shear strain equations [11-13] are -41.50 and $-24.84 \%$, respectively.

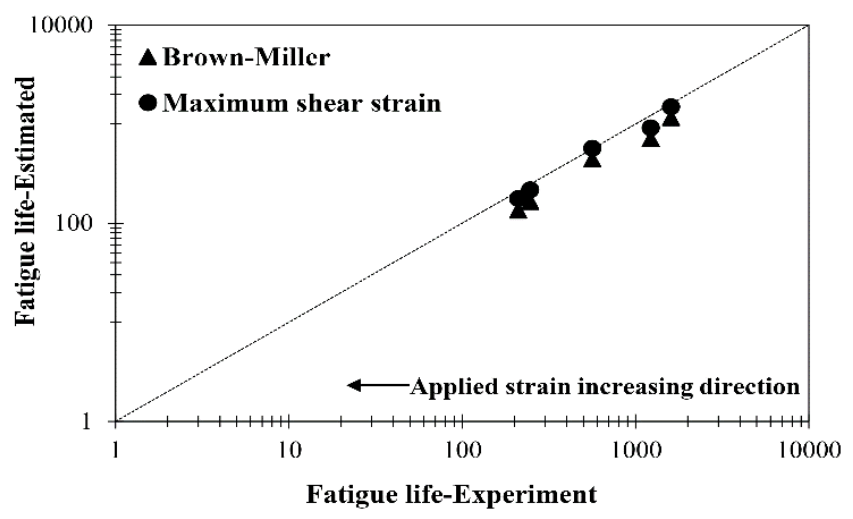

Fig. 6. A comparison between the predicted and the experimental LCF lifetimes.

Table 3. The percent error between the numerical and experimental low cycle fatigue.

\begin{tabular}{|c|c|c|c|c|c|}
\hline \multirow{2}{*}{$\begin{array}{c}\text { Multiaxial strain-life } \\
\text { criterion }\end{array}$} & \multicolumn{5}{|c|}{ Percent error for each strain amplitude (\%) } \\
\cline { 2 - 6 } & $\mathbf{\pm 0 . 5}$ & $\pm \mathbf{0 . 6}$ & $\pm \mathbf{0 . 7 5}$ & $\pm \mathbf{1 . 0}$ & $\pm \mathbf{1 . 2}$ \\
\hline Brown \& Miller & -27.27 & -41.50 & -21.17 & -32.79 & -35.71 \\
\hline Maximum shear strain & -6.12 & -24.84 & 1.96 & -11.74 & -15.72 \\
\hline
\end{tabular}

\subsection{Effect of surface irregularities on the fatigue life}

One of the major problems, which has aroused the interest of researchers for decades, is the influence of surface machinability on the material strength. This section investigates the effect of surface defects induced by machining process on the fatigue life of $316 \mathrm{SS}$, at room temperature. The maximum shear strain criteria with Morrow mean stress correction [12-13] 
has been used herein as it provides accurate fatigue life estimation as indicated in the previous section.

To examine the influence of surface quality on the low cycle fatigue using Fe-Safe software [15], different values of the surface roughness average $R_{a}$ have been considered, namely $\mathrm{R}_{\mathrm{a}} \leq 0.25 \mu \mathrm{m}, 0.25<\mathrm{R}_{\mathrm{a}} \leq 0.6 \mu \mathrm{m}, 0.6<\mathrm{R}_{\mathrm{a}} \leq 1.6 \mu \mathrm{m}, 1.6<\mathrm{R}_{\mathrm{a}} \leq 4 \mu \mathrm{m}, 4<\mathrm{R}_{\mathrm{a}}$ $\leq 16 \mu \mathrm{m}, 16<\mathrm{R}_{\mathrm{a}} \leq 40 \mu \mathrm{m}$, and $40<\mathrm{R}_{\mathrm{a}} \leq 75 \mu \mathrm{m}$, for strain amplitudes ranging between \pm 0.5 and $\pm 1.2 \%$. Figure 7 illustrates the obtained strain-life curves, with different surface roughness values, for cylindrical specimens, subjected to various strain amplitudes. As observed, the low cycle fatigue life increases as the applied strain amplitude and the surface roughness average increase, which further prove the accuracy of the numerical simulation.

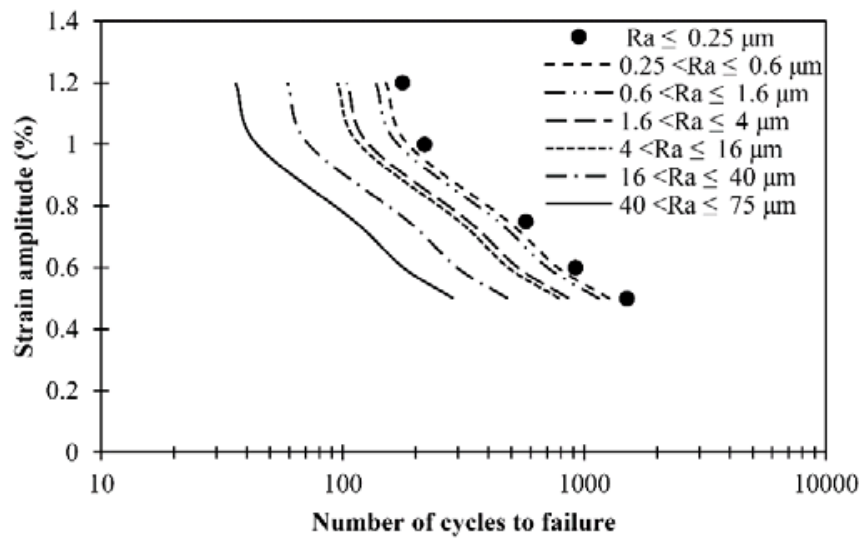

Fig.7. Strain-life curves of $316 \mathrm{SS}$ at ambient temperature, for different surface roughness average values.

Table 4 illustrates the percentage reduction in the low cycle fatigue life with respect to the fatigue lives for samples with $\mathrm{R}_{\mathrm{a}} \leq 0.25 \mu \mathrm{m}$, which are assumed the true value. It is clear from this table that the low fatigue life is strongly dependent on the machining process. For example, when $40<\mathrm{R}_{\mathrm{a}} \leq 75 \mu \mathrm{m}$, the average percentage reduction of the fatigue life is $-80.30 \%$, while it is only $-15.24 \%$ for $0.25<\mathrm{R}_{\mathrm{a}} \leq 0.6 \mu \mathrm{m}$. The main reason behind that is poor machinability generates surface defects, which are considered as small cracks or notches on the surface $[9,18]$. Hence, stress concentration phenomenon and localized multiaxial stress modes arise near the surface defects, which boosts the fatigue crack to initiate and provokes eventually a shorter fatigue life. It can therefore be concluded that a good surface treatment is typically required to increase the durability of mechanical components in service.

Table 4. Percentage reduction in the fatigue life as a function of the surface roughness average value and strain amplitude.

\begin{tabular}{|c|c|c|c|c|c|c|}
\hline $\begin{array}{c}\text { Strain } \\
\text { amplitude } \\
\mathbf{( \% )}\end{array}$ & \multicolumn{5}{|c|}{ Percentage Reduction for each Surface Roughness Average Value $\boldsymbol{R}_{\boldsymbol{a}}(\boldsymbol{\mu m})$} \\
\cline { 2 - 7 } & $\mathbf{0 . 2 5}<\boldsymbol{R}_{\boldsymbol{a}} \leq \mathbf{0 . 6}$ & $\mathbf{0 . 6}<\boldsymbol{R}_{\boldsymbol{a}} \leq \mathbf{1 . 6}$ & $\begin{array}{c}\mathbf{0 . 6}<\boldsymbol{R}_{\boldsymbol{a}} \leq \\
\mathbf{1 . 6}\end{array}$ & $\mathbf{1 . 6}<\boldsymbol{R}_{\boldsymbol{a}} \leq \mathbf{4}$ & $\mathbf{1 6}<\boldsymbol{R}_{\boldsymbol{a}} \leq \mathbf{4 0}$ & $\mathbf{4 0}<\boldsymbol{R}_{\boldsymbol{a}} \leq \mathbf{7 5}$ \\
\hline $\mathbf{0 . 5}$ & -15.63 & -23.67 & -42.55 & -47.54 & -67.95 & -80.92 \\
\hline $\mathbf{0 . 6}$ & -15.65 & -23.48 & -42.28 & -47.39 & -67.72 & -80.65 \\
\hline$\pm \mathbf{0 . 7 5}$ & -15.53 & -23.39 & -42.06 & -46.95 & -67.36 & -80.45 \\
\hline$\pm \mathbf{1 . 0}$ & -14.68 & -22.48 & -41.28 & -46.33 & -66.97 & -79.82 \\
\hline$\pm \mathbf{1 . 2}$ & -14.69 & -22.60 & -41.24 & -46.33 & -66.67 & -79.66 \\
\hline
\end{tabular}




\section{Conclusions}

In the present study, the low cycle fatigue behavior was numerically investigated using ABAQUS software, for cylindrical specimens made of 316 Stainless Steel subjected to nominal strain amplitude ranging from \pm 0.5 to $\pm 1.2 \%$, at room temperature. Furthermore, the fatigue life was estimated using the maximum shear strain as well as Brown-Miller multiaxial strain-life criteria on Fe-Safe software. Various surface roughness average values were considered in this study to analyze the effect of surface quality on fatigue life. The following are the overall conclusions:

- The numerically obtained first cycle hysteresis loop for all strain amplitudes was in good agreement with that obtained experimentally.

- Compared to the experimental low cycle fatigue lives, it was revealed that the multiaxial strain-life criteria, namely the maximum shear strain as well as Brown-Miller both with Morrow mean stress effect provided conservative fatigue life estimation. Hence, they can be used to predict the low cycle fatigue of smooth specimens made of $316 \mathrm{SS}$, subjected to fully reversed uniaxial tension-compression cyclic loading.

- Since the maximum shear strain criterion provided more accurate fatigue life estimation than the Brown-Miller criterion, it was used to assess the effluence of surface imperfections, resulting from the machining process, on the low cycle fatigue of mechanical components. It was found that the numerical simulation is reliable and that poor machinability has a great effect on the material durability.

\section{References}

1. Dassault Systemes Simulia Corp, Safe Technology Limited, 2 (2016).

2. J.Büttner and R. Keen, U Chi Press Journals, 02 (2011).

3. O.H.Basquin, In Proc Am Soc Test Mater, 10, 625-630 (1910).

4. F.Coffin, Trans ASME, 76, 931-950 (1954).

5. S.S.Manson, Washington, D.C: NACA, (1954).

6. A. Nussbaumer, L.Borges, L.Davaine, John Wiley \& Sons, (2012).

7. NA.Alang, NA.Razak, AK.Miskam, Int. J. Eng. Technol, 160-163, (2011).

8. P.J.Maziasz, and J.T.Busby, ORNL, TN US, (2012).

9. I.Abarkan, R.Shamass, Z.Achegaf, and A.Khamlichi, J. Press. Vessel Technol, (2020).

10. Abaqus 6.16. Product of Dassault Systemes Simulia Corp, Providence, 2016.

11. M. W. Brown, and K. J.Miller, Proc. Inst. Mech. Eng, 187,745-755 (1973).

12. X.Chen, Sh.Xu, and D. Huang, FFEMS, 22, 679-686 (1999).

13. J.Morrow, Adv. Eng, 04, 21-9 (1968).

14. B. Joadder, J. Shit, S. Acharyya, and S. Dhar, MSA, 02, 1730-1740, (2011).

15. Fe-Safe 6.2, Product of Dassault Systemes Simulia Corp, Providence, (2016).

16. J. Lemaitre, J.L. Chaboche, CUP, Cambridge, (1990).

17. P. L. Menezes, Kishore, and S. V. Kailas, Scratching of Materials and Applications, 262-279 (2006).

18. D. Taylor and O. M. Clancy, FFEMS, 14, 329-336 (1991). 